\title{
Determination of Micro Impurity Element Potassium in Sodium Metavanadate by ICP-OES
}

\author{
Yong CHENG \\ PanGang Group Research Institute Co., Ltd., State Key Laboratory of Vanadium and Titanium \\ Resources Comprehensive Utilization, Panzhihua 617000, Sichuan, China \\ luck2005_1@sina.com
}

\begin{abstract}
Keywords: Inductively Coupled Plasma Atomic Emission Spectrometry, ICP-OES, Sodium Metavanadate, Potassium, Microanalysis, Matrix Effects.
\end{abstract}

\begin{abstract}
The sodium metavanadate samples are digested completely by hydrochloric acid and nitric acid $\left(\mathrm{V}_{\mathrm{HCl}}: \mathrm{V}_{\mathrm{HNO} 3}=5: 1\right)$, and then made a directly determination of micro element potassium of 0.010 to $1.0 \%$ by inductively coupled plasma atomic emission spectrometry (ICP-OES). In the coexistence medium of $42 \%$ vanadium and $20 \%$ sodium, the method systematically investigated the matrix effects, spectral interferences, background effects and other interference factors, and a concluded was made: the matrix elements vanadium and sodium do not produce spectral interference in the determination of micro potassium, the matrix effect of vanadium and background noise enhanced the detection signal of potassium spectral lines, however, the matrix effects of sodium weakened the signal of potassium. In the high concentrations of vanadium and sodium coexistence medium system, the overall matrix effect still raised up the detection signal of potassium analysis lines and the background noise. Therefore, the test have taken the preferred analysis spectral lines, background correction region and ICP spectrometer parameters and other testing measures, and through comprehensive application of the matrix matching and synchronization background correction methods to eliminate interference in determination of the micro potassium that caused by the high content of vanadium and sodium coexist matrix. The results show: Detection limit is $480 \mu \mathrm{g} / \mathrm{L}(766.490 \mathrm{~nm})$ or $480 \mu \mathrm{g} / \mathrm{L}$ (769.896nm); Background equivalent concentration is $200 \mu \mathrm{g} / \mathrm{L}(766.490 \mathrm{~nm})$ or $210 \mu \mathrm{g} / \mathrm{L}$ $(769.896 \mathrm{~nm})$; RSD $\leq 2.72 \%(766.490 \mathrm{~nm})$ or $\mathrm{RSD} \leq 3.60 \%(769.896 \mathrm{~nm})$; Recovery rate is between $95.0 \%$ and $107.5 \%$.
\end{abstract}

\section{Introduction}

Sodium metavanadate is an important vanadium fine chemical product, which is widely used in the industrial field. It not only can be used as chemical reagents, catalysts, driers and mordant, etc., but also could be used as basic chemical raw materials for the production of ammonium metavanadate or potassium metavanadate and other products. The content of the micro impurity potassium is one of the key indicators affecting product's quality control, at present, papers of the analysis methods that determination of sodium metavanadate chemical composition have not retrieved, and only have a small number of similar detection methods reported in the literatures[1-3] that determination of sodium, potassium, phosphorus, arsenic, iron in the vanadium pentoxide or ammonium metavanadate samples by atomic absorption spectroscopy or inductively coupled plasma atomic emission spectrometry. However, the existing F-AAS or ICP-OES methods are mainly applied to the determination of the vanadium pentoxide or other mere vanadium-containing matrix samples. The detection conditions and performance indicators and other important technical parameters of existing methods do not fully consider these impact factors caused by the high concentration of alkali metal sodium element. The sodium metavanadate coexist $42 \%$ vanadium and $20 \%$ sodium, so the original method cannot be applied to such a complex matrix media.

In this paper, the samples are digested by hydrochloric acid and nitric acid, and then made a direct determination of micro impurity element potassium by ICP-OES in the sodium metavanadate. The 
complex system in the coexistence of about $42 \%$ vanadium and $20 \%$ sodium was the tested and summarized from the spectral interferences, matrix effects, background noise and other interference factors on the determination. This method take the preferred analysis wavelength, background correction area, as well as instrument operating parameters and other detection conditions, at the same time, combined application matrix matching and synchronization background correction two technical measures, therefore to overcome the high vanadium and high sodium coexistence matrix, argon molecular band and other interference factors impact on the determination of micro potassium. The method has less interference factors, wider detection range, less steps, convenient and easy to control, short inspection cycle, excellent technical performance and other characteristics, it can meet the need for a direct determination of 0.010 to $1.0 \%$ of the micro impurity potassium in the sodium metavanadate.

\section{Experimental}

\section{Instruments and Reagents}

iCAP 6300 full spectrum of direct-reading plasma atomic emission spectrometer, Elix water purifier.

Superior grade hydrochloric acid and nitric acid.

Potassium standard stock solution, whose concentration is $1.0000 \mathrm{mg} / \mathrm{mL}$.

\section{Working Conditions of Instrument}

ICP spectrometer working conditions: RF power is $800 \mathrm{~W}$, auxiliary gas flow rate (Ar) is $1.5 \mathrm{~L} / \mathrm{min}$, nebulizer pressure is $0.26 \mathrm{MPa}$, peristaltic pump speed is $55 \mathrm{r} / \mathrm{min}$, observation height is $11.0 \mathrm{~mm}$, each measurement need integration 3 times and each integration time is $15 \mathrm{~s}$.

\section{Test Method}

$0.5000 \mathrm{~g}$ sodium metavanadate sample was Weighted and put into a $250 \mathrm{~mL}$ conical flask, rinse bottle wall and disperse samples with $10 \mathrm{~mL}$ water, then add $10 \mathrm{~mL}$ hydrochloric acid and until the digestion reaction is completed at a low temperature heating conditions, add $2 \mathrm{~mL}$ nitric acid and boiled to produce a uniform large bubbles, the test solution after cooling is diluted with water in $100 \mathrm{~mL}$ volumetric flask. And the test solution is measured in plasma atomic emission spectrometer according to the working conditions of instrument in paragraph 1.2.

\section{The Calibration Curve}

According to the proportion of sodium metavanadate as $42 \%$ vanadium and $20 \%$ sodium, the appropriate amount of high purity vanadium pentoxide and sodium chloride are digested in accordance with the 1.3 test methods, then this pure matrix reagent solution used as blank correction and matrix matching bottoming solution, then added standard solution of potassium and formulated into a series of different concentrations matrix matching calibration curve whose matrix composed are entirely consistent with the sodium metavanadate sample solution. The potassium element content covered the concentration range of $0.010 \%$ to $1.0 \%$.

\section{Results and Discussion}

\section{Spectral Interference Test and the Selection of Spectral Lines}

The emission spectrometry of potassium atomic is very simple and its spectral lines are not too much, so there is not too much choice to select the analytical line for the determination of micro potassium. Interference tests showed that the two high sensitivity analysis lines of the K $766.490 \mathrm{~nm}$ and $\mathrm{K} 769.896 \mathrm{~nm}$ are not interfered by matrix elements vanadium and sodium, however, in the scanning window of these two spectral lines, a flat signal baseline produced by the blank solution of 
$42 \%$ vanadium with its intensity is approximately 4 times stronger than the signal baseline of the digestion reagent blank, indicates that the vanadium matrix effects strengthen the signal of potassium analysis line. The mixed solution with $42 \%$ vanadium and $20 \%$ sodium that is similar to the sodium metavanadate matrix components, still enjoys a straight signal baseline , and its strength was only approximately 2 times than the reagent blank, which is significantly lower than blank background signal that only enjoys the same concentration of vanadium, in other words, the signal intensities drop down about half while added into $20 \%$ sodium, which indicates that alkali metal sodium element which is easily producing ionized and producing a strong inhibitory effect on potassium analysis line, as a result, in the coexistence medium of high concentrations of vanadium and sodium, we can not ignore the strong impact on the determination that caused by the vanadium and sodium matrix elements.

Since the wavelengths of K 766.490nm and K 769.896nm near infrared region, the argon molecular bands produce strong background noise and lead to the significantly higher background baseline. The signal baseline were relatively flat, and the net signal intensity values and the background equivalent concentration value and the background equivalent concentration value are consistent with the digestion reagent blank, which indicates that the matrix matching and synchronization background correction can effectively eliminate its impact as showed in Fig.1.

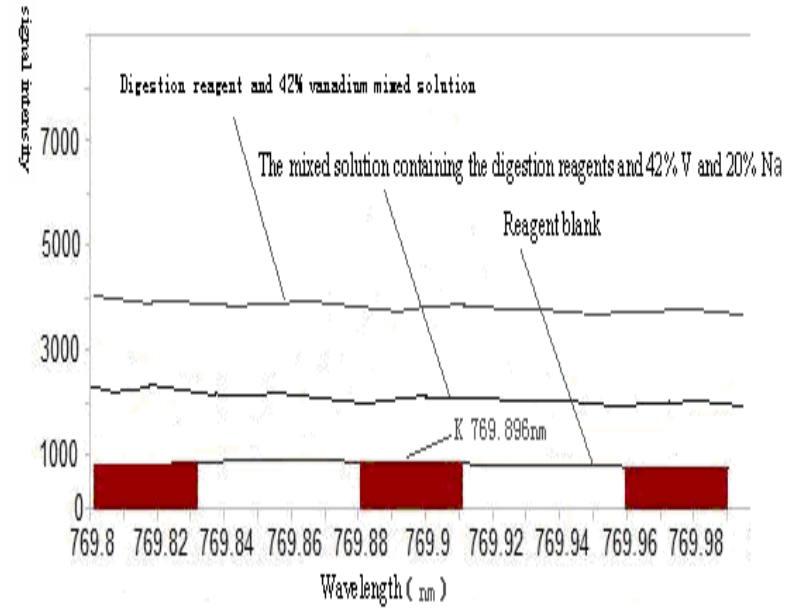

Fig.1-1 Matrix effect of $\mathrm{Na}$ and $\mathrm{V}$ test chart

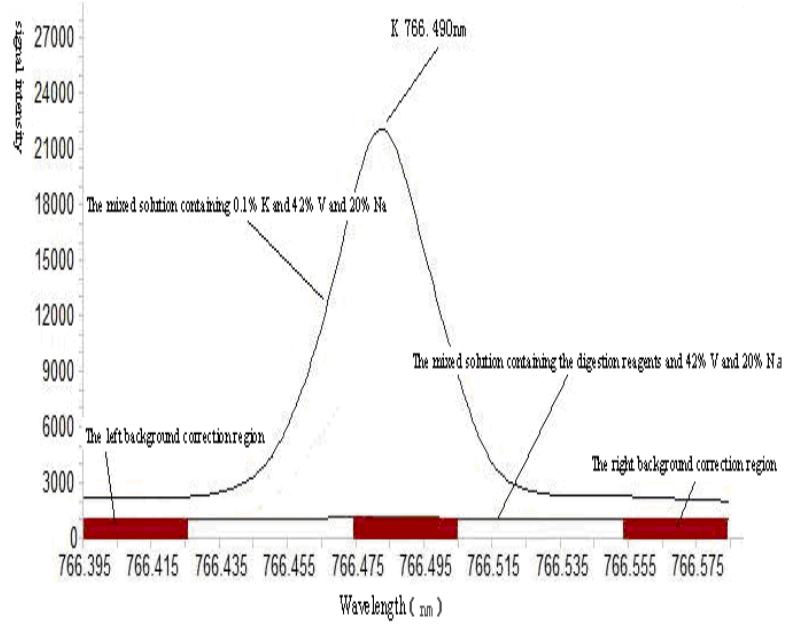

Fig.1-2 K 766.490nm interference test chart

According to the above tests, the preferred potassium analysis wavelength and background correction position are shown in Tab.1.

Tab.1 The element analysis wavelength and the background correct position (nm)

\begin{tabular}{ccc}
\hline $\begin{array}{c}\text { element } \\
\text { Wavelength }\end{array}$ & $\begin{array}{c}\text { the left background correct } \\
\text { position }\end{array}$ & $\begin{array}{c}\text { the right background correct } \\
\text { position }\end{array}$ \\
\hline K 766.490 & $766.395 \sim 766.425$ & $766.545 \sim 766.575$ \\
K 769.896 & $769.800 \sim 769.820$ & $769.970 \sim 770.000$ \\
\hline
\end{tabular}

\section{Background Equivalent Concentration and Detection Limit}

In order to eliminate a strong impact on the determination of micro potassium that caused by sodium metavanadate matrix, the calibration curve (1.4) have to match by matrix according to the contents of vanadium and sodium in sodium metavanadate, as a resoult to make the consistent of the calibration curve and the composition of test solution. In accordance with the proportion of $42 \% \mathrm{~V}$ and $20 \% \mathrm{Na}$, the purity vanadium pentoxide and sodium chloride were digested and prepared into the substrate reagent blank solution by the test method of 1.3. The blank solution was paralleling measured 11 times continuously; along with the concentration value corresponding to 10 times the standard deviation was used as the lower detection limit of the method. Background equivalent 
concentration $(\mathrm{BEC})=20$ blank standard deviation $\div(20$ blank relative standard deviation $\times$ the slope of the standard curve). The results are shown in Tab.2.

Tab.2 The background equivalent concentration and the element Detection limit(\%)

\begin{tabular}{ccc}
\hline Wavelength & BEC & Detection limits \\
\hline K 766.490nm & 0.0040 & 0.0096 \\
K 769.896nm & 0.0042 & 0.0092 \\
\hline
\end{tabular}

As seen from Tab.2, BEC of the method is $0.0040 \sim 0.0042 \%(200 \sim 210 \mu \mathrm{g} / \mathrm{L})$, so it caused weak effect on the detection results, and it indicates that the impact of reagent background and high vanadium high sodium coexist matrix are effectively eliminated by matrix matching and synchronization background correction measures. The detection limit is $0.0092 \sim 0.0096 \%$ (460 $480 \mu \mathrm{g} / \mathrm{L}$ ) and meet the needs of detection of micro potassium.

\section{The Linear Relationship of the Calibration Curve and Its Detection Range Test}

The standard solutions of calibration curve are prepared in accordance with 1.4, and made curve fitting and linear regression. The results showed that the linear relationship is very good in the concentration range of 0.010 to $1.0 \%$, with the correlation coefficient $r>0.999$ 3, shown in Fig. 2 .

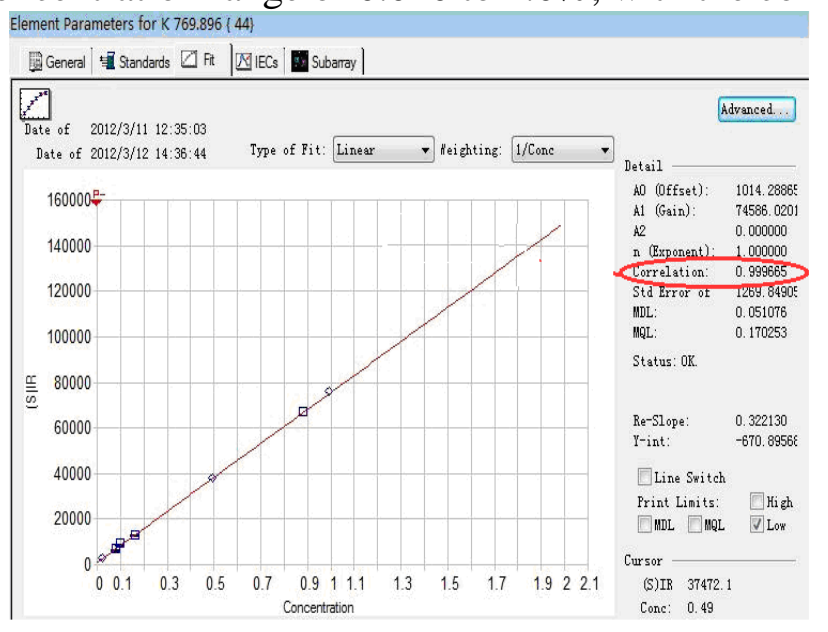

Fig.2-1 K 766.896 nm calibration curve

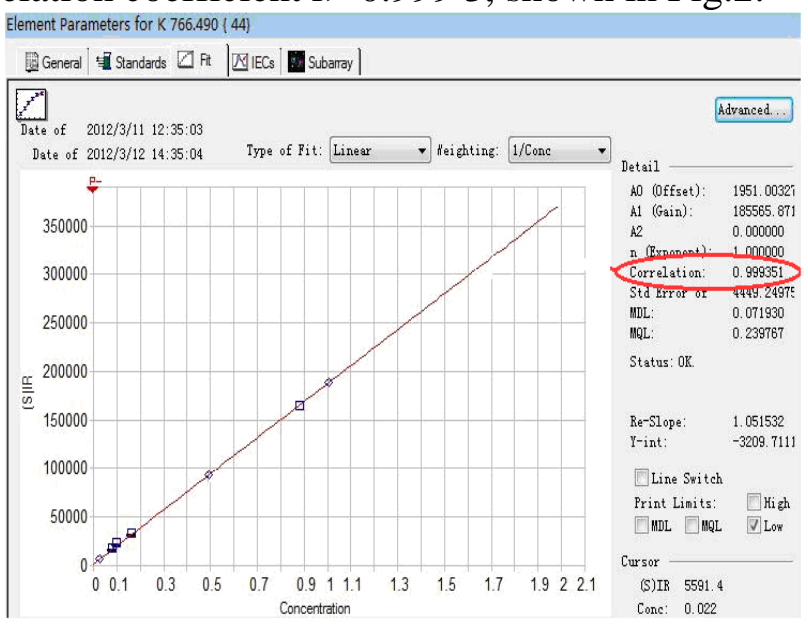

Fig.2-2 K 766.490nm calibration curve

\section{The Precision Test}

Tab.3 The precision experiments results of method $(n=8)$

\begin{tabular}{ccccc}
\hline & \multicolumn{2}{c}{ Sample 1 } & \multicolumn{2}{c}{ Sample 2 } \\
\cline { 2 - 5 } & K 766.490nm & K 769.896nm & K 766.490nm & K 769.896nm \\
\hline 1 & 0.1835 & 0.1644 & 0.2795 & 0.2573 \\
2 & 0.1809 & 0.1644 & 0.2809 & 0.2615 \\
3 & 0.1825 & 0.1616 & 0.2817 & 0.2641 \\
4 & 0.1764 & 0.1649 & 0.2923 & 0.2877 \\
5 & 0.186 & 0.1573 & 0.2918 & 0.2694 \\
6 & 0.1862 & 0.165 & 0.2829 & 0.2703 \\
7 & 0.1784 & 0.1596 & 0.2797 & 0.2621 \\
8 & 0.172 & 0.1573 & 0.2918 & 0.2604 \\
Average (W/\%) & 0.1807 & 0.1618 & 0.2851 & 0.2666 \\
Standard deviation & 0.0049 & 0.0034 & 0.0058 & 0.0096 \\
RSD (\%) & 2.72 & 2.07 & 2.04 & 3.60 \\
\hline
\end{tabular}


Two sodium metavanadate samples were select randomly, and each sample was separately prepared into eight test solutions. Each solution was measured and the eight test results' relative standard deviation were calculated. The precision of the method are shown in Tab.3.

Tab.3 shows that: the RSD $\leq 2.72 \%(766.490 \mathrm{~nm}), \mathrm{RSD} \leq 3.60 \%(769.896 \mathrm{~nm})$, which indicate that the method is accurate and reliable.

\section{Recovery of the Method Test}

The actual sodium metavanadate samples are analyzed in accordance with the experimental methods and the spike recovery is tested, the results are shown in Tab.4.

In Tab.4, the recovery is between $95.0 \%$ and $107.5 \%$, which indicates the results of the method are accurate and reliable.

Tab.4 Recovery test results(\%)

\begin{tabular}{lccccccccc}
\hline \multirow{2}{*}{ Wavelength } & \multicolumn{4}{c}{ Sample 3 } & \multicolumn{3}{c}{ Sample 4 } \\
\cline { 2 - 9 } & $\begin{array}{c}\text { Found before } \\
\text { add standard }\end{array}$ & Added & $\begin{array}{c}\text { Found after } \\
\text { add standard }\end{array}$ & $\begin{array}{c}\text { Recovery } \\
\text { Found after } \\
\text { add standard }\end{array}$ & Added & $\begin{array}{c}\text { Found after } \\
\text { add standar }\end{array}$ & Recovery \\
\hline K 766.490nm & 0.0135 & 0.0100 & 0.0238 & 103.0 & 0.0066 & 0.0040 & 0.0109 & 107.5 \\
\hline K 769.896nm & 0.0116 & 0.0100 & 0.0211 & 95.0 & 0.0055 & 0.0040 & 0.0094 & 97.5 \\
\hline
\end{tabular}

\section{Conclusions}

This method is used for the direct determination of micro impurity element potassium of the sodium metavanadate, the quantitative detection range is ranged from $0.010 \%$ to $1.0 \%$. In this paper, the spectral interferences, matrix effects, background noise and other interference factors in the complex coexistence system of high vanadium high sodium has tested and summarized, preferred analysis spectral lines, background correction region and ICP parameters and other measures has been selected, and combined with the utilization of the matrix matching and synchronization background correction methods, the impact of high vanadium and high sodium matrix and argon molecular bands on the determination of micro potassium has been effectively solved. The technical performance of the method is excellent, it is simple and practical, easy to operate, with less interference factors and lower detection limitation and background equivalent concentration, wide detection range, good precision, high accuracy and other characteristics.

\section{References}

[1]Ferrous Metallurgy Industry Standards of the People's Republic of China [S] YB/T 5335-2006. Vanadium Pentoxide-Determination of sodium oxide and potaiumss oxide content-The flame atomic absorption spectrometric method.

[2]Liu GuoHong. Simultaneous determination of $\mathrm{Al}, \mathrm{Fe}, \mathrm{Ca}, \mathrm{As}, \mathrm{Na}, \mathrm{K}, \mathrm{Si}$ in the ammonium metavanadate by ICP-OES [J]. Western China Science and Technology, 2011.10 (22): 19-20.

[3]Li Tao, Feng Ziping, Chen Jingfa, Yulian, inductively coupled plasma emission spectrometry determination of vanadium content in the ammonium metavanadate [J]. Physical Testing And Chemical Analysis Part B: Chemical Analysis, 2011.(09):1086-1087. 